\title{
Nosocomial Infections in a Medical-Surgical Intensive Care Unit
}

\author{
Nasser Yehia A. Aly ${ }^{a}$ b Haifaa H. Al-Mousa ${ }^{b}$ El Sayed M. Al Asar ${ }^{c}$ \\ ${ }^{a}$ Department of Tropical Medicine and Hygiene, Faculty of Medicine, University of Alexandria, Alexandria, Egypt; \\ ${ }^{b}$ Directorate of Infection Control and ' Department of Microbiology, Farwaniya Hospital, Ministry of Health, Kuwait
}

\author{
Key Words \\ Nosocomial infection · Intensive care unit • \\ Device-associated infection
}

\begin{abstract}
Objective: We aimed to describe the pattern of nosocomial infections in an adult medical-surgical intensive care unit (ICU). Subjects and Methods: A 2-year prospective cohort study of nosocomial infection surveillance in a 15-bed adult combined medical and surgical ICU of Farwaniya Hospital, Kuwait, was carried out. Data were collected between January 2004 and December 2005 using the standard surveillance protocols and nosocomial infection site definitions of the National Nosocomial Infections Surveillance System's ICU surveillance component. Results: Of 1,173 patients hospitalized in the ICU for an aggregate duration of 6,855 days, 89 patients acquired a total of 140 nosocomial infections; 46 (33\%) ventilator-associated pneumonia (VAP), 33 (24\%) central-line-associated bloodstream infection and 15 (11\%) catheter-associated urinary tract infection, 22 (16\%) cutaneous infection and 24 (17\%) other infections. The overall patient day rate was $20.6 / 1,000$ patient days. The patient infection rate was $10.6 / 100$ patients at risk. The mean VAP rate was $9.1 / 1,000$ ventilator days $(95 \% \mathrm{Cl}, 5-13.2)$, the central-line-associated bloodstream infection rate 5.5/1,000 central line days $(95 \% \mathrm{Cl}, 3.2-7.8)$ and the catheter-associated urinary tract infection rate $2.3 / 1,000$ catheter days $(95 \% \mathrm{Cl}, 1.2-3.4)$. Of all nosocomial infections, 119 (85\%) were culture-con-
\end{abstract}

firmed and 21 (15\%) were clinically defined culture-negative infections. Of the culture-confirmed nosocomial infections, 81 (68\%) were Gram-negative, 32 (27\%) Gram-positive and 6 (5\%) fungal. The most frequent organism was Pseudomonas aeruginosa (20,17\%), followed by Acinetobacter baumannii $(15,13 \%)$, Klebsiella spp. (13, 11\%) and Escherichia coli (10, 8\%). The crude mortality was $27 \%$ among ICU-infected patients. Conclusion: VAP was the most common nosocomial infection in our ICU. Gram-negative organisms were more commonly reported as etiologic agents of ICU infections.

Copyright $\odot 2008$ S. Karger AG, Basel

\section{Introduction}

Nosocomial infections have been recognized for over a century as a critical problem affecting the quality of health care and a principal source of adverse health care outcomes [1]. Intensive-care-unit (ICU)-acquired infection rates are 5-10 times higher than hospital-acquired infection rates in general ward patients [2]. This increased risk of nosocomial infection results from 3 major factors: (a) intrinsic risk factors related to the need for intensive care; (b) invasive medical devices, and (c) crowding and animate reservoirs that increase the risk of cross infection in the ICU [3]. Such infections continue to complicate the clinical course of critically ill patients and consequently to create substantial economic and human costs. ICUs are an area of considerable antibiotic use in

\section{KARGER}

Fax +4161306 1234

E-Mail karger@karger.ch

www.karger.com
(C) 2008 S. Karger AG, Basel

1011-7571/08/0175-0373\$24.50/0

Accessible online at:

www.karger.com/mpp
Nasser Yehia A. Aly

Department of Infection Control, Farwaniya Hospital

Ministry of Health, PO Box 12414

71655 Al-Shamiya (Kuwait)

Tel. +965 488 8000, ext. 3816, Fax +965 488 2684, E-Mail nasseryehia@gmail.com 
which antibiotic-resistant organisms are prevalent [4] and patients are prone to secondary infections after exposure to broad-spectrum antimicrobials [5].

Surveillance for nosocomial infections is the cornerstone of prevention and control of these infections [6]. Furthermore, one of the purposes of surveillance is to benchmark with other units or to determine the impact of eventual interventions targeted at the prevention of nosocomial infections. Among many nation-wide surveillance systems (KISS in Germany, CLIN in France or $\mathrm{NIH}$ in the UK), the National Nosocomial Infections Surveillance System (NNIS) remains the most representative data source on nosocomial infection rates. Risk adjustment is provided by stratifying the data by type of ICU and type of invasive medical device and by presenting the infection rate as infections per 1,000 device days [7]. The objective of this study was to conduct nosocomial infection surveillance and measure device-associated infection rates, crude mortality, and the microbiological and antibiotic resistance profiles of infecting pathogens in the main ICU of Farwaniya Hospital, Kuwait.

\section{Subjects and Methods}

A 2-year prospective cohort study of nosocomial infection surveillance was carried out in a 15-bed adult combined medical and surgical ICU of Farwaniya Hospital, Kuwait. Neither medical nor surgical patients represented more than $80 \%$ of the total subjects in the unit. The hospital is a 650 -bed multispecialty acute care facility. It serves an area with a total population of about 750,000 .

Infection surveillance was implemented for all patients admitted to the ICU with a stay longer than $48 \mathrm{~h}$ during the period January 1, 2004, to December 31, 2005. The Center for Disease Control definitions of nosocomial infections [8] and the standard surveillance methods of NNIS were used, as previously described [9]. The overall patient infection rate was calculated as the number of all infections per 100 patients at risk, the overall patient day rate as the number of all infections per 1,000 patient days and the device-associated infection rates as the number of infections per 1,000 device days. The average length of stay (ALOS) was a proxy for infection risk and was calculated using the equation below:

$$
\operatorname{ALOS}=\frac{\mathrm{d}}{\mathrm{c}+\frac{\mathrm{a}}{2}-\frac{\mathrm{b}}{2}}
$$

where $a$ is the number of patients in the ICU on the first day of the month, $b$ indicates the number of patients in the ICU on the first day of the next month, $\mathrm{c}$ is the number of patients admitted to the ICU during the month and $d$ the number of days spent by all patients in the ICU during that month. The adjusted rate by ALOS is the quotient of dividing the patient infection rate by ALOS.

Bacteria were cultured using standard microbiological methods [10]. They were identified by conventional methods according
Table 1. Demographic characteristics of ICU-infected patients

\begin{tabular}{|c|c|}
\hline Demographic characteristics & Patients \\
\hline \multicolumn{2}{|l|}{ Age } \\
\hline$<20$ years & $9(10)$ \\
\hline $20-40$ years & $21(24)$ \\
\hline $40-60$ years & $40(45)$ \\
\hline$>60$ years & $19(21)$ \\
\hline \multicolumn{2}{|l|}{ Gender } \\
\hline Male & $66(74)$ \\
\hline Female & $23(26)$ \\
\hline \multicolumn{2}{|l|}{ Nationality } \\
\hline Kuwaiti & $33(37)$ \\
\hline Non-Kuwaiti & $56(63)$ \\
\hline \multicolumn{2}{|l|}{ Admission diagnosis } \\
\hline RTA/multiple trauma & $34(38.2)$ \\
\hline Acute cerebrovascular accidents & $18(20.2)$ \\
\hline Acute myocardial infarction & $8(9)$ \\
\hline Acute abdominal emergency & $7(7.9)$ \\
\hline Acute pulmonary edema & $5(5.6)$ \\
\hline Respiratory failure & $4(4.5)$ \\
\hline Status epilepticus & $3(3.4)$ \\
\hline Sepsis & $3(3.4)$ \\
\hline Upper gastrointestinal hemorrhage & $2(2.2)$ \\
\hline Tetanus & $2(2.2)$ \\
\hline Drug overdose & $2(2.2)$ \\
\hline Acute pylonephritis & $1(1.1)$ \\
\hline \multicolumn{2}{|l|}{ Outcome } \\
\hline Survived & $65(73)$ \\
\hline Expired & $24(27)$ \\
\hline Total & $89(100)$ \\
\hline
\end{tabular}

Figures in parentheses are percentages. RTA $=$ Road traffic accident.

to the Kirby-Bauer disc diffusion technique [11] and the VITEK 2 system (bioMérieux, France).

Statistical analysis was performed with the $\chi^{2}$ test, odds ratio and, where appropriate, bivariate correlation using the Pearson correlation coefficient. The $p$ value was set at $0.05 \%$. Summary statistics for continuous or ordinal variables were expressed as means and standard deviations and medians with percentiles and discrete variables as percentages. The data were analyzed using SPSS for Windows version 11 (SPSS Inc., Chicago, Ill., USA).

\section{Results}

Of 1,173 patients, 89 developed 140 nosocomial infections. The overall patient day rate was $20.6 / 1,000$ patient days. The overall patient infection rate was $10.6 / 100$ patients at risk. The mean age was $45 \pm 19$ years. Thirtythree patients (37\%) were Kuwaiti and 56 (63\%) were of 
Table 2. Means and percentiles of device-associated infection rates and device utilization ratios compared to NNIS figures

\begin{tabular}{|c|c|c|c|c|c|c|c|}
\hline & & Mean & Perc & ntile & & & \\
\hline & & & 10 & 25 & $\begin{array}{l}50 \\
\text { (median) }\end{array}$ & 75 & 90 \\
\hline Device-assc & iated infec & tion $r$ & & & & & \\
\hline CA-UTI & KNNIS & 2.3 & 0 & 0 & 1.3 & 3.9 & 6.7 \\
\hline & NNIS & 3.9 & 1.3 & 2.1 & 3.3 & 5.2 & 7.5 \\
\hline CLA-BSI & KNNIS & 5.5 & 0 & 0 & 4.1 & 8.6 & 15.4 \\
\hline & NNIS & 4 & 1.7 & 2.6 & 3.4 & 5.1 & 7.6 \\
\hline VAP & KNNIS & 9.1 & 0 & 0.98 & 5.1 & 12.5 & 28.9 \\
\hline & NNIS & 5.4 & 1.2 & 2.6 & 4.6 & 7.2 & 9.9 \\
\hline Device utiliz & mon ralle & & & & & & \\
\hline Urinary & KNNIS & 0.95 & 0.91 & 0.93 & 0.96 & 0.97 & 0.98 \\
\hline catheter & NNIS & 0.78 & 0.65 & 0.74 & 0.82 & 0.87 & 0.90 \\
\hline Central line & KNNIS & 0.88 & 0.78 & 0.83 & 0.88 & 0.93 & 0.96 \\
\hline & NNIS & 0.57 & 0.36 & 0.47 & 0.56 & 0.66 & 0.74 \\
\hline Ventilator & KNNIS & 0.78 & 0.68 & 0.75 & 0.79 & 0.82 & 0.87 \\
\hline & NNIS & 0.43 & 0.23 & 0.32 & 0.43 & 0.55 & 0.62 \\
\hline
\end{tabular}

KNNIS = Kuwait NNIS in the studied ICU.

other nationalities. The main admission diagnoses were multiple trauma/road traffic accident: 34 (38.2\%) and acute cerebrovascular accidents: 18 (20.2\%). The demographic characteristics of ICU-infected patients are given in table 1.

The distribution of infections was as follows: ventilator-associated pneumonia (VAP): 46, central-line-associated bloodstream infection (CLA-BSI): 33 and catheter-associated urinary tract infection (CA-UTI): 15 . The remaining infections were cutaneous/subcutaneous infections: 22 , lower respiratory tract other than pneumonia: 10 , surgical site infections: 6 , upper respiratory tract infections: 3 , eye: 3 , and ear: 2 .

The mean CA-UTI rate was $2.3 / 1,000$ catheter days, the CLA-BSI rate 5.5/1,000 central line days and the VAP rate 9.1/1,000 ventilator days. Of 6,855 patient days, the device utilization ratio was 0.95 for urinary catheters, 0.88 for central line and 0.78 for ventilator utilization (table 2). The ALOS was 7.8 days and the adjusted patient infection rate by ALOS was 1.4.

The mean overall patient infection rate was strongly correlated with the length of stay $(\mathrm{r}=0.549, \mathrm{p}=0.006)$ and ventilator use $(r=0.497, p=0.013)$. The CLA-BSI, VAP and CA-UTI did not correlate with length of stay $(\mathrm{r}=-0.217$, $-0.093,0.068 ; p=0.308,0.667,0.752$, respectively) or with device-associated utilization $(\mathrm{r}=-0.298,0.219,0.033 ; \mathrm{p}=$ $0.157,0.304,0.878$, respectively, for each device).

Nosocomial Infections in an Intensive Care Unit
Table 3. Microbial etiology of culture-confirmed infections in the ICU

\begin{tabular}{lc}
\hline Organism & Cases \\
\hline Gram-negative (n = 81) & \\
Pseudomonas aeruginosa & 20 \\
Acinetobacter baumannii & 15 \\
Klebsiella pneumoniae & 12 \\
Escherichia coli & 10 \\
Haemophilus influenzae & 6 \\
Enterobacter cloacae & 5 \\
Stenotrophomonas maltophilia & 4 \\
Morganella morganii & 3 \\
Klebsiella oxytoca & 1 \\
Moraxella catarrhalis & 1 \\
Proteus mirabilis & 1 \\
Providencia stuartii & 1 \\
Pseudomonas fluorescens & 1 \\
Serratia marcescens & 1 \\
Gram-positive (n = 32) & \\
Enterococcus fecalis & 8 \\
Staphylococcus aureus & 7 \\
Coagulase-negative staphylococci & 6 \\
MRSA & 4 \\
Listeria monocytogenes & 2 \\
Streptococcus pneumoniae & 2 \\
Streptococcus pyogenes & 1 \\
Streptococcus viridans & 1 \\
Diphtheroids & 1 \\
Fungal (n = 6) & \\
Candida albicans & 6 \\
\hline Total & \\
\hline & \\
\hline
\end{tabular}

Coagulase-negative staphylococci: Staphylococcus epidermidis $(\mathrm{n}=3)$; Staphylococcus hominis $(\mathrm{n}=2)$ and other coagulasenegative staphylococci $(\mathrm{n}=1)$. MRSA = Methicillin-resistant Staphylococcus aureus.

Of all nosocomial infections, 119 (85\%) were cultureconfirmed and 21 (15\%) were clinically defined culturenegative infections. Of the culture-confirmed nosocomial infections, 81 (68\%) were Gram-negative, 32 (27\%) Gram-positive and 6 (5\%) fungal. Among the 119 organism shown in table 3 , the most frequently isolated was Pseudomonas aeruginosa: 20 (17\%); Acinetobacter baumannii: 15 (13\%); Klebsiella spp.: 13 (11\%) and Escherichia coli: 10 (8\%). Interestingly, $2 / 10(20 \%)$ of the E. coli isolates and 2/13 (15\%) Klebsiella spp. tested positive for extended-spectrum $\beta$-lactamase.

Overall, 70\% of the Gram-negative isolates were susceptible to ciprofloxacin, $96 \%$ to meropenem, $70 \%$ to ceftazidime, $83 \%$ to cefotaxime, $45 \%$ to piperacillin, $83 \%$ to amikacin and $68 \%$ to gentamycin. With regard to Gram- 
positives, all Staphylococcus aureus isolates were sensitive to oxacillin and gentamycin. Methicillin-resistant $S$. aureus, S. epidermidis and enterococci were all sensitive to vancomycin.

Of all 1,173 patients admitted to the ICU, 268 (23\%) died. Of the 1,084 without nosocomial infections, 244 (23\%) died compared to 24 of 89 (27\%) with nosocomial infections. There was no association between nosocomial infection and overall mortality in the ICU $(p=0.405)$. Notably, mortality among patients with ICU-acquired infections was related to an ICU stay $\geq 6$ days (odds ratio $=2.7, \mathrm{CI}=1.2-5.9)$.

\section{Discussion}

Patients in ICUs are at high risk for nosocomial infections and since 1987 have been monitored in the NNIS by site-specific, risk-adjusted infection rates according to ICU type [12]. The risk-adjusted benchmark infection rates and device utilization ratios are published annually for use by both NNIS and non-NNIS hospitals [12].

The predominant types of infection and the ecology of potential pathogens vary between different ICU types and among similar types. This may be a result of factors such as patient case mix, device utilization rates, teaching affiliation and empirical antibiotic usage patterns. Different groups such as trauma patients have unique predispositions to different infections. There is a wide distribution of device utilization rates and thus infection rates among these patient groups [13]. Device-associated infection rates are the best available rates for comparisons because they are not confounded by length of stay, total number of hospital beds or differences in the associated device utilization [14].

A lower rate of CA-UTI was noted in our ICU than in any type of NNIS-participating ICUs. Our median CAUTI rate (50th percentile) corresponded to the 10th percentile of NNIS. Interestingly, our rate did not reflect lower rates of urinary catheter use. On the contrary, we found that the urinary catheter utilization ratio in our ICU was a 'high outlier'. This finding could be related to adequate antibiotic coverage, diversity in microbial etiology and differences in susceptibility to antibiotic between our ICU and NNIS-participating ICUs. Variations in the severity of illness among catheterized patients and in the duration of catheterization might have in part contributed to this discrepant result [15].

On the other hand, the median for CLA-BSI lies between the 50th and 75th percentile and the median cen- tral line utilization was above the 90th percentile of NNIS [7]. A similar observation was recorded for VAP and ventilator utilization. This value denotes that between 50 and $75 \%$ of similar NNIS ICUs had lower rates than ours. Furthermore, the 2 device utilization ratios were 'high outliers'. Since central lines and ventilators are significant risk factors for BSI and VAP, efforts should be targeted to reduce their use or limit the duration of their use.

Gram-negative bacilli are frequently associated with nosocomial infections in ICU patients, particularly VAP and CA-UTI [16]. The results of this study are in accord with that finding and showed that of all Gram-negative organisms $(\mathrm{n}=81)$, P. aeruginosa accounts for $(25 \%), A$. baumannii for $19 \%$, Klebsiella spp. for $16 \%$ and E. coli for $12 \%$. In a previous survey of the prevalence and antibiotic susceptibility pattern of consecutive Gram-negative bacterial isolates in 2 ICUs in Saudi Arabia (Jeddah) and Kuwait, the most common bacterial isolates in the Kuwait ICU were P. aeruginosa (26\%), Acinetobacter spp. (33\%) Klebsiella pneumoniae (17\%) and E. coli (3\%) compared to $26,9,20$ and $23 \%$ of the same organisms in Jeddah ICUs [17].

The emergence of antimicrobial-resistant pathogens in ICUs has made treating ICU-acquired infections very difficult and, in some cases, impossible. A summary of the rates of resistance for the most common pathogens associated with nosocomial infections among ICU patients has been previously reviewed [18]. The observed percentage of isolates positive for extended-spectrum $\beta$ lactamase in our ICU reflects one of the emerging resistance patterns.

Compared with an earlier report from Kuwait [17], our results of antibiotic susceptibility patterns show increased resistance to ciprofloxacin and piperacillin among Gram-negative bacteria, possibly due to their widespread use, but a similar sensitivity pattern to carbapenem antibiotics.

Previous reports from Western countries indicate that extensive use of the $\beta$-lactam and fluoroquinolone drugs has contributed to the rapid emergence of multidrug-resistant Gram-negative bacteria, particularly Klebsiella spp., Enterobacter spp., Pseudomonas spp. and Acinetobacter spp. $[19,20]$. The crude mortality among our patients $(27 \%)$ was similar to that reported by Bueno-Cavanillas et al. [21] (28\%) and Ylipalosaari et al. [22] (25.7\%) but lower than that reported by Girou et al. [23] (58.5\%). In general, the differences reported between studies may be related to some confusion between the associated and attributable parts. The impact of ICU infections on hospital mortality is controversial. However, recent reports 
support the conclusion that nosocomial infections increase the risk of death in critically ill patients [21,22]. In this study, there was no difference in mortality between nosocomially infected and noninfected patients $(\mathrm{p}<$ $0.05)$. It seems likely that mortality was judged by the severity of the underlying disease or condition, which was often complicated and life-threatening.

The surveillance data have some limitations. The study included major infection sites, device utilization, and types of organisms and their susceptibility to commonly used antibacterial agents but did not attempt to investigate the underlying disease conditions of patients or the role of nosocomial infection. Viral infections may be underreported because appropriate cultures were not always performed. The study was a single-center investigation, which limits its generalizability. Nevertheless, it establishes a benchmark for future studies.

\section{Conclusion}

Gram-negative organisms were the most commonly reported etiologic agents of ICU infections. The median CA-UTI rate in the ICU of this study was lower than that of similar US ICUs of the NNIS. However, the median VAP and CLA-BSI rates, though somewhat higher, were still comparable to NNIS figures. Infection control measures should be targeted at these infections.

\section{Acknowledgments}

The authors thank Mrs. Suja Thomas, RN, and Rehana Ikram, $\mathrm{RN}$, from the Department of Infection Control for their contribution to the collection of surveillance data, and the ICU staff at Farwaniya Hospital for their cooperation.

\section{References}

1 Gaynes RP, Horan TC: Surveillance of nosocomial infections; in Mayhall CG (ed): Hospital Epidemiology and Infection Control, ed 3. Philadelphia, Lippincott Williams \& Wilkins, 1999, pp 1285-1318.

$\checkmark 2$ Weinstein RA: Nosocomial infection update. Emerg Infect Dis 1998;4:416-420.

-3 Weber DJ, Raasch R, Pharm D, Rutala WA: Nosocomial Infections in the ICU: the growing importance of antibiotic-resistant pathogens. Chest 1999;115:S34-S41.

-4 Albrich WC, Angstwurm M, Bader L, Gartner R: Drug resistance in intensive care units. Infection 1999;27(suppl 2):S19-S23.

5 Eggimann P, Pittet D: Infection control in the ICU. Chest 2001;120:2059-2093.

-6 Arya SC, Agarwal N, Agarwal S, George S, Singh K: Nosocomial infection: hospital infection surveillance and control. J Hosp Infect 2004;58:242-243.

7 National Nosocomial Infections Surveillance System: National Nosocomial Infections Surveillance (NNIS) System Report: data summary from January 1992 through June 2004, issued October 2004. Am J Infect Control 2004;32:470-485.

8 Horan TC, Gaynes RP: Surveillance of nosocomial infections; in Mayhall CG (ed): Hospital Epidemiology and Infection Control, ed 3. Philadelphia, Lippincott Williams \& Wilkins, 2004, pp 1659-1702.

$\checkmark 9$ Emori TG, Culver DH, Horan TC, Jarvis WR, White JW, Olson DR, Banerjee S, Edwards JR, Martone WJ, Gaynes RP, Hughes JM: National Nosocomial Infections Surveillance (NNIS) System: Description of surveillance methodology. Am J Infect Control 1991;19:19-35.
10 Barrow GI, Feltham RKA: Cowan and Steel's Manual for the Identification of Medical Bacteria, ed 3. Cambridge, Cambridge University Press, 1993.

11 National Committee for Clinical Laboratory Standards: Performance Standards for Antimicrobial Disc Susceptibility Tests. Wayne, National Community for Clinical Laboratory Standards, 2000, NCCLS document, M2-A7.

12 National Nosocomial Infections Surveillance System: Nosocomial infection rates for interhospital comparison: limitations and possible solutions. Infect Control Hosp Epidemiol 1991;12:609-612.

13 Richard M, Thursky K, Buising K: Epidemiology, prevalence and sites of infections in intensive care units. Semin Respir Crit Care Med 2003;24:3-22.

14 Richards MJ, Edwards JR, Culver DH, Gaynes RP; National Nosocomial Infections Surveillance System: Nosocomial infections in pediatric intensive care units in the United States. Pediatrics 1999;103:1-7.

15 Roberts JA, Fussekk EN, Kaack MB: Bacterial adherence to urethral catheters. J Urol 1990;144:264-269.

16 Fridkin SK, Welbel SF, Weinstein RA: Magnitude and prevention of nosocomial infections in the intensive care unit. Infect Dis Clin North Am 1996;11:479-496.
17 Rotimi VO, al-Sweih NA, Feteih J: The prevalence and antibiotic susceptibility pattern of Gram-negative bacterial isolates in two ICUs in Saudi Arabia and Kuwait. Diagn Microbiol Infect Dis 1998;30:53-59.

18 Fridkin SK, Gaynes RP: Antimicrobial resistance in intensive care units. Clin Chest Med 1999;20:303-316.

19 Ballow CH, Schentag JH: Trends in antibiotic utilization and bacterial resistance: report of the National Nosocomial Resistance Surveillance group. Diagn Microbiol Infect Dis 1992;15(suppl 2):S375-S425.

20 Pierson CL, Friedman BA: Comparison of susceptibility to beta-lactam antimicrobial agents among bacteria isolated from intensive care units. Diagn Microbiol Infect Dis 1992;15(suppl 2):S19-S30.

-21 Bueno-Cavanillas A, Delgado-Rodriguez M, Lopez-Luque A, Schaffino-Cano S, GalvezVargas R: Influence of nosocomial infection on mortality rate in an intensive care unit. Crit Care Med 1994;22:55-60.

22 Ylipalosaari P, Ala-Kokko TI, Laurila J, Ohtonen P, Syrjälä1 H: Intensive care acquired infection is an independent risk factor for hospital mortality: a prospective cohort study. Crit Care 2006;10:R66.

23 Girou E, Stephan F, Novara A, Safar M, Fagon JY: Risk factors and outcome of nosocomial infections: results of a matched casecontrol study of ICU patients. Am J Respir Crit Care Med 1998;157:1151-1158. 